\title{
UNA NUEVA ESPECIE DEL GÉNERO BATHYSCIOLA JEANNEL, 1910 DE LOS PIRINEOS CENTRALES, ESPAÑA (COLEOPTERA, LEIODIDAE, CHOLEVINAE, LEPTODIRINI)
}

\author{
J. Fresneda* y J. Comas**
}

\begin{abstract}
RESUMEN
Se describe una nueva especie del género Bathysciola Jeannel, 1910 -Bathysciola fadriquei n. sp.- perteneciente al grupo schiodtei; ésta ha sido hallada en medio subterráneo profundo, en dos cuevas situadas en Sierra de Bernera, macizo del Bisaurín, Pirineo de Huesca, Aragón, España. Los caracteres diferenciales se encuentran básicamente en las estructuras genitales: la nueva especie se caracteriza por presentar en el complejo faneroide del saco interno del edeago unas faneras apicales muy grandes, triangulares, fuertemente esclerotizadas, con un lóbulo exterior asociado. Se discute la posición taxonómica. Se completa el estudio con ilustraciones de las estructuras que permiten su diferenciación, clave de identificación y datos de distribución.
\end{abstract}

Palabras clave: Coleoptera, Leiodidae, Cholevinae, Leptodirini, Bathysciola fadriquei n. sp., Pirineos centrales, España.

\begin{abstract}
A new species of the genus Bathysciola Jeannel, 1910 from the Central Pyrenees (Coleoptera, Leiodidae, Cholevinae, Leptodirini)

A new species of the genus Bathysciola Jeannel, 1910 -Bathysciola fadriquei n. sp.is described in the schiodtei group. The species was collected in a subterranean environment, in two caves in Sierra de Bernera, Bisaurín massif, Pyrenees of Huesca, Aragón, Spain. The most relevant characters of the genital structures were examined in detail: the new species is characterized by the complex phanerae of the internal sac of the aedeagus, with very large, triangular and strongly sclerotised apical phanerae presenting an associated external lobe. The taxonomic position of the new species is discussed. The study is completed with figures of the most characteristic structures, an identification key and distribution data.
\end{abstract}

Key words: Coleoptera, Leiodidae, Cholevinae, Leptodirini, Bathysciola fadriquei n. sp., Central Pyrenees, Spain. 


\section{Introducción}

En el transcurso de las exploraciones llevadas a cabo por espeleólogos de la Secció d'Investigacions Espeleologiques del Centre Excursionista Áliga de Barcelona, se visitaron distintas cavidades subterráneas de la Sierra de Bernera (comarca de la Jacetania, Aragón); en dos de ellas se recolectó, al rastreo y mediante trampas de caída, una serie de Leptodirini Lacordaire, 1854 del género Bathysciola Jeannel, 1910 pertenecientes al grupo schiod$t e i$. Dadas las características morfológicas $\mathrm{y}$ anatómicas observadas, particularmente las presentadas por el edeago y concretamente su saco interno, estos ejemplares no se han podido adscribir a ninguna de las especies previamente conocidas, perteneciendo pues a un nuevo taxon desconocido para la ciencia que se describe en este artículo.

El género Bathysciola fue establecido por R. Jeannel en 1910 que designó como su especie tipo a Bathyscia aubei Kiesenwetter, 1850. Sin embargo hoy día también se considera perteneciente al mismo género la especie descrita con el nombre Catops pusillus Motschulsky, 1840, especie tipo de Catopsinus Motschulsky, 1870, género actualmente considerado sinónimo de Bathysciola. Así pues, Catopsinus tiene prioridad sobre Bathysciola. Sin embargo, el género representado por el nombre Bathysciola, que ha sido el más usado, reúne un numerosísimo grupo de especies que presenta una dilatada distribución: desde los Pirineos hasta el Cáucaso; este hecho es inusual entre los Leptodirini y por lo tanto si alguna vez se realizase un trabajo de revisión del género, es probable que éste fuese fragmentado y que tanto el nombre Catopsinus como el de Bathysciola se conservasen. En ausencia de análisis cladístico que demostrase la heterogeneidad de Bathysciola, pero basándose en un buen conocimiento de la morfología y anatomía de sus especies, esta necesidad de revisión la han manifestado Jeannel (1910), Giachino (1998), Newton (1998), Perreau (2000) y Fresneda y Salgado $(2000,2006)$. Fresneda et al. (2007) ya reconocen en Bathysciola el conjunto para-polifilético que sospechaban los autores anteriores. El grupo schiodtei de Bathysciola, fue propuesto por Perreau (2000) y redefinido por Fresneda \& Salgado (2006). Se han descrito doce táxones de los que Fresneda \& Salgado (2006) reconocen nueve como válidos. Habita básicamente en ambas vertientes de la mitad occidental del macizo pirenaico, aunque también se sabe de su presencia en las cercanías de Burdeos, y en la región de Calvados, al norte de Francia. De las especies conocidas, cuatro se encuentran exclusivamente en la vertiente sur de los Pirineos, en los relieves geográficamente ibéricos.

\section{Resultados}

\section{Bathysciola fadriquei n. sp.}

SERIE TIPO. Holotipo $\sigma^{7}$ de Huesca, Jaca, Aragüés del Puerto, Cueva de Lizara, UTM: X 694400-Y 4737375-Z $1.470 \mathrm{~m}$ (datum europeo de 1950), en trampa de caída del 12-IV-2006 al 10-IX-2006, F. Fadrique leg. Depositado en la colección Comas (Barcelona). Paratipos: $11 \sigma^{7} \sigma^{7}$ y 10 우 우 de la misma localidad y fechas que el holotipo; $10^{\pi}$ y 1 ㅇ de la misma localidad, 12-IV-2006, F. Fadrique leg.; 4 우 우 de la misma localidad, en trampas de caída del 27-III-2005 al 12-IV-2006, F. Fadrique leg. $10^{7}$ de Huesca, Jaca, Aragüés del Puerto, Espelunga de Mediodía, 25-III-2005, UTM: X 695050-Y 4738619-Z 1.904 m (datum europeo de 1950), F. Fadrique leg. Los paratipos se encuentran depositados en las colecciones Comas (Barcelona), Fresneda (Llesp, Lleida), Salgado (León), Museo Nacional de Ciencias Naturales de Madrid y en la del Museu de Ciències Naturals (Zoologia) de Barcelona.

DESCRIPCIÓN DEL HOLOTIPO. La longitud medida desde el borde anterior del pronoto hasta el ápice de los élitros es de 1,33 mm. Esta especie es anoftalma y despigmentada, con el cuerpo de contorno oval, regularmente menguante hacia atrás, y con el pronoto más convexo que los élitros, aunque éstos tomados en conjunto también son marcadamente convexos; la pubescencia es amarillenta, corta, densa y yacente en toda la superficie; no existe ángulo pronoto-elitral.

Las antenas sobrepasan un poco los ángulos posteriores del pronoto; su longitud es de $0,62 \mathrm{~mm}$; el séptimo artejo es grueso aunque su longitud es el doble de su anchura, el octavo es de aspecto redondeado, pero oblongo, y los de la maza son escasamente más largos que anchos, excepto el undécimo cuya longitud es dos veces su anchura.

El pronoto mide $0,45 \mathrm{~mm}$ de largo por $0,73 \mathrm{~mm}$ de ancho; es tan ancho como la base de los élitros, con los márgenes laterales regularmente curvados en toda su longitud; la anchura máxima se encuentra situada en la base, que es marcadamente sinuosa; los ángulos posteriores son agudos con el vértice romo; el tegumento está marcado por puntos algo granulosos densamente dispuestos, lo que le confiere un aspecto mate.

Los élitros tienen la anchura máxima situada en el tercio basal, muy cerca de la base; los márgenes laterales están regularmente arqueados y el ápice elitral es ampliamente redondeado; se puede observar un fino reborde marginal; el tegumento se encuentra marcado por estriolas transversas. 

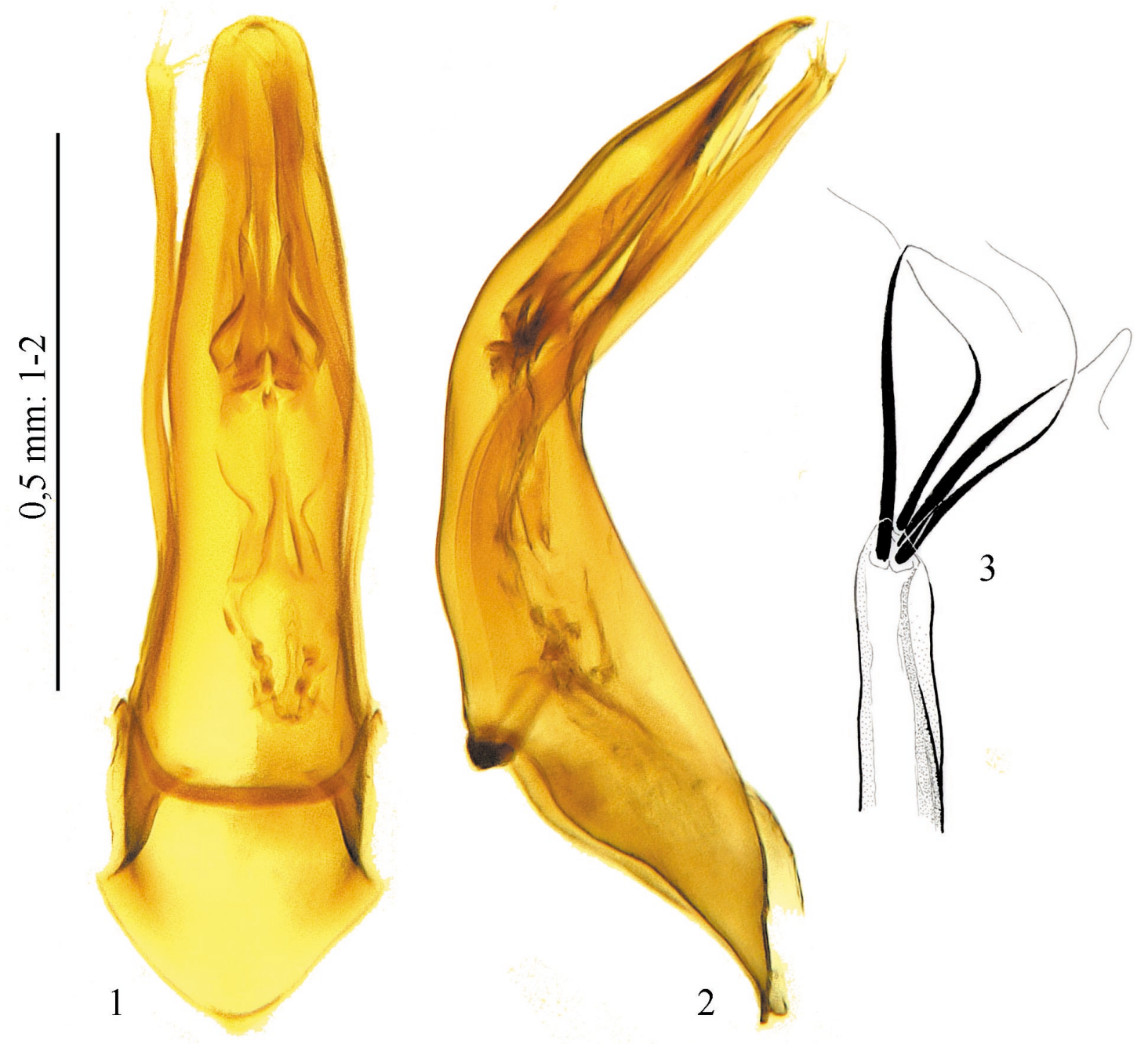

Figs. 1-3.-Bathysciola fadriquei n. sp. 1) edeago en visión dorsal. 2) edeago en visión lateral. 3) ampliación del ápice del estilo lateral.

Figs. 1-3.- Bathysciola fadriquei n. sp. 1) aedeagus in dorsal view. 2) aedeagus in lateral view 3) detailed view of the apical region of the lateral style.

La quilla mesosternal es alta, con el margen anterior fuertemente redondeado y un diminuto dentículo situado en el margen ventral en las cercanías del vértice.

Las patas son proporcionalmente largas y finas, con las mesotibias un poco curvadas y las metatibias rectas; los protarsos son pentámeros y están dilatados aunque el primer artejo, que es el más ancho, es un poco más estrecho que el extremo distal de la protibia; el metafémur no presenta espina femoral.

Genitalia masculina. El lóbulo medio del edeago en visión lateral (Fig. 2) está doblado en ángulo obtuso aproximadamente por la mitad; la mitad basal es muy robusta y la cara dorsal es fuertemente sinuosa en toda su longitud; en visión dorsal (Fig. 1) tiene el sector terminal manifiestamente estrechado, con el ápice redondeado. Los estilos laterales son tan largos como el lóbulo medio y tienen el ápice (Fig. 3) provisto de cuatro largas sedas sinuosas de longitud similar. El saco interno del edeago (Fig. 4) está dividido en tres regiones; en la región basal la pieza en Y la forman dos varillas simétricas muy finas que llevan en la punta un nódulo grueso con forma de haba y articulado por 

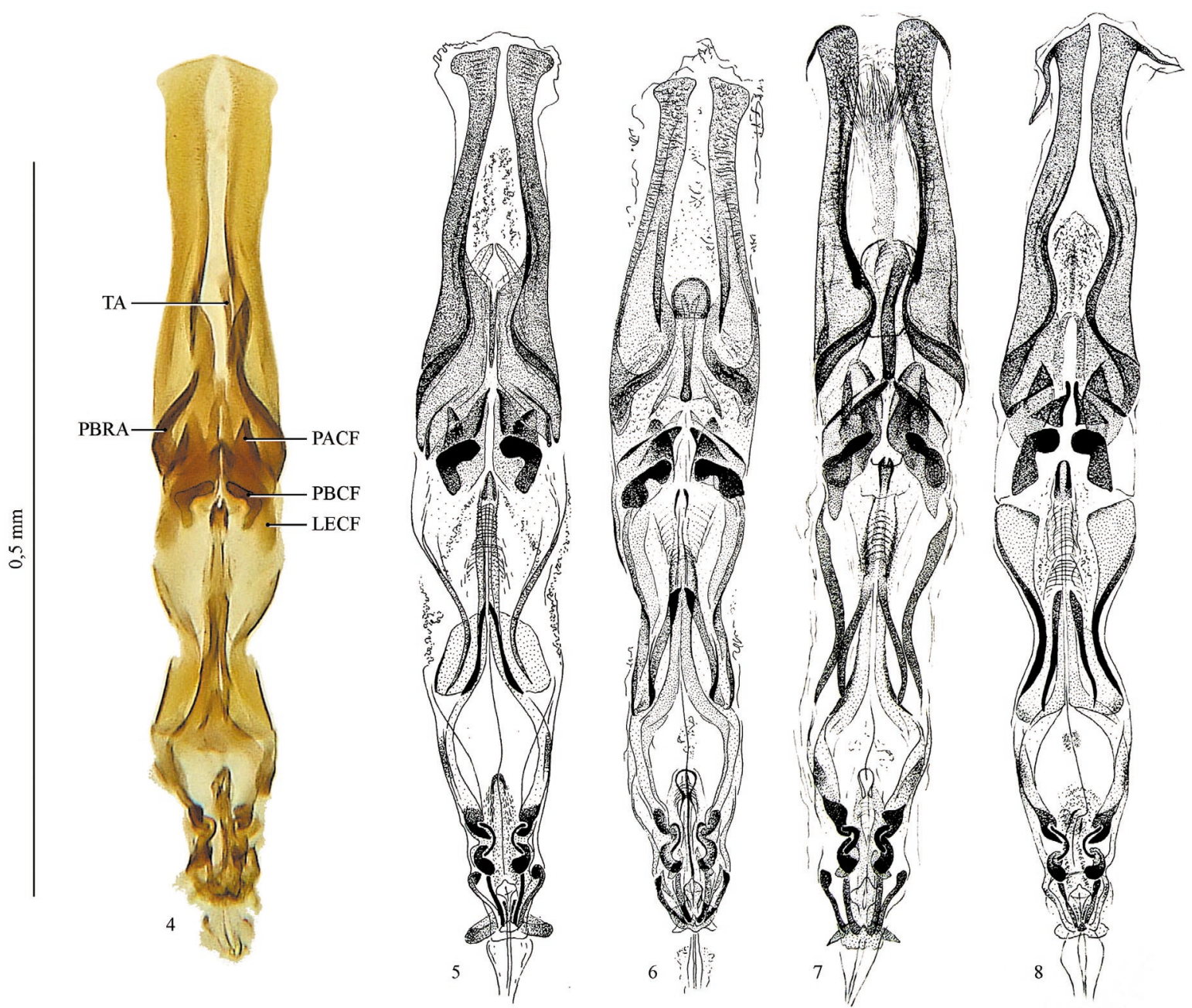

Figs. 4-8.- Saco interno del edeago en visión dorsal de 4) Bathysciola fadriquei n. sp. 5) B. breuili. 6) B. diegoi. 7) B. obermaieri. 8) B. rugosa. LECF, lóbulos exteriores del complejo faneroide; PACF, pieza apical del complejo faneroide; PBCF, pieza basal del complejo faneroide; PBRA, pedúnculos de las bandas de refuerzo apical; TA, túbulo apical.

Figs. 4-8.- Internal sac of the aedeagus in dorsal view of 4) Bathysciola fadriquei n. sp. 5) B. breuili. 6) B. diegoi. 7) B. obermaieri. 8) B. rugosa. LECF, exterior lobes of the phanerae complex; PACF, apical piece of the phanerae complex; PBCF, basal piece of the phanerae complex; PBRA, stalks of the apical reinforcement bands; TA, apical tube.

medio de un túbulo con otro nódulo de igual forma; se encuentran faneras anexas a la pieza en Y; en la región media existen faneras ventrales, dorsales y dos más exteriores, que discurren por ambos lados; en el extremo superior de la región media existe un complejo formado por tres faneras muy esclerotizadas y articuladas entre sí: las basales están dobladas por la mitad formando un ángulo recto y van acompañadas de unos lóbulos exteriores; las apicales son muy grandes, triangulares y están fuertemente esclerotizadas; en la región apical las bandas de refuerzo se ensanchan en el sector basal formando unas láminas que casi entran en contacto en el eje de simetría del saco, y en la cara interna de su parte inferior existen sendos pedúnculos que rodean por la parte exterior el complejo faneroide; entre las bandas de refuerzo se encuentra un túbulo.

DiAGNOSIS DE LA HEMBRA. Las hembras son algo más pequeñas y estrechas que los machos. La longitud media de los paratipos hembra es de $1,22 \mathrm{~mm}$, y de 1,24 mm en los machos. Los protarsos no están 
dilatados y son tetrámeros como es normal en los Leptodirini (sensu Perreau, 2000: excepto Sciaphyes Jeannel, 1910). La espina ventral del octavo esternito abdominal es larga y de forma triangular, con la base ancha (Fig. 9).

Complejo espermatecal. La espermateca (Fig. 10) tiene la región media rectilínea, delgada e hialina; los dos lóbulos están diferenciados, y casi forman un ángulo recto respecto al plano de la región media; el lóbulo basal es más grande y alargado que el apical, que es esférico; el conducto espermático es unas 4-5 veces más largo que la espermateca siendo más delgado cuanto más cerca de ésta y formando un engrosamiento localizado poco antes del punto de inserción con la espermateca; en las proximidades de la bolsa copulatriz atraviesa un disco esclerotizado; la glándula espermática desemboca en el mismo punto de inserción del conducto espermático con la espermateca.

EtimologíA. La especie está dedicada a su recolector, F. Fadrique, asíduo colaborador del Museu de Ciències Naturals (Zoologia) de Barcelona.

\section{Discusión}

La nueva especie de Bathysciola que se describe pertenece al grupo schiodtei, nombre propuesto por Perreau (2000) para las especies de la sección VI de Jeannel (1924); basándose en las estructuras genitales de ambos sexos, aunque dando especial relevancia al saco interno del edeago, el grupo fue redefinido por Fresneda \& Salgado (2006), que incluyeron en este grupo nueve táxones válidos. Se caracteriza -incluyendo la nueva especie- por presentar el saco interno del edeago dividido en tres regiones; en la región basal la pieza en Y la forman dos varillas simétricas muy finas que llevan en la punta un nódulo grueso con forma de haba que se articula por medio de un túbulo con otro nódulo de igual forma; se encuentran faneras anexas a la pieza en Y; en la región media existen faneras ventrales, dorsales y dos más exteriores, que discurren por ambas zonas laterales y son muy largas; en el extremo superior de la región media se encuentra el complejo faneroide («phanerae complex» de Fresneda \& Salgado, 2006) formado por tres faneras articuladas entre sí; en la región apical las bandas de refuerzo tienen el extremo basal grueso y fuertemente esclerotizado.

Bathysciola fadriquei n. sp. presenta estructuras genitales que se encuentran también en otras especies incluso de distribución norpirenaica:
- las piezas basales del complejo faneroide acodadas se encuentran también en las especies norpirenaicas B. bigerrica Jeannel, 1930, B. grandis (Fairmaire, 1856) y B. parallela (Jeannel, 1907), y en las surpirenaicas $B$. breuili Bolívar, 1921 y B. rugosa (Sharp, 1872).

- el túbulo entre las bandas de refuerzo apical se encuentra también en las especies norpirenaicas $B$. bigerrica, $B$. grandis y $B$. parallela, y en las surpirenaicas $B$. diegoi Salgado y Fresneda, 2001 y B. obermaieri Bolívar, 1918 (el más similar).

- en la cara interna de la parte inferior de las bandas de refuerzo apical existen sendos pedúnculos fuertemente esclerotizados también en las especies norpirenaicas $B$. bigerrica y $B$. parallela (el más similar); también existen, aunque son menos evidentes, en la especie norpirenaica $B$. fauveli Jeannel, 1924 y en las surpirenaicas $B$. breuili, B. obermaieri y B. rugosa.

Resulta evidente que estas estructuras son útiles para identificar las especies, pero no se ha encontrado un patrón de distribución coherente; por este motivo se cree que su valor filogenético debe ser más bien escaso y debería ser estudiado en un contexto más amplio. Así pues, se ha optado por comparar la nueva especie con las que habitan su misma región en la vertiente sur de los Pirineos ( $B$. breui$l i, B$. diegoi, B. obermaieri y B. rugosa), dado que es más probable que esté más relacionada con ellas que con las norpirenaicas.

Las diferencias más llamativas que se pueden observar son:

- Las piezas basales del complejo faneroide del saco interno del edeago son acodadas en Bathysciola fadriquei n. sp. (Fig. 4: РBCF), B. breuili (Fig. 5) y B. rugosa (Fig. 8), mientras que son rectas en $B$. diegoi (Fig. 6) y B. obermaieri (Fig. 7).

- Bathysciola fadriquei n. sp. tiene un túbulo esclerotizado entre las bandas de refuerzo apical (Fig. 4: TA) que también se encuentra en $B$. diegoi (Fig. 6) y B. obermaieri (Fig. 7), pero no existe en B. breuili (Fig. 5) ni en B. rugosa (Fig. 8 ); además los machos de esta última especie son muy grandes, con los élitros muy anchos y deprimidos, más pequeños, con los élitros regularmente menguantes desde la base y convexos en $B$. fadriquei n. sp.

- Bathysciola fadriquei n. sp. tiene unos pedúnculos fuertemente esclerotizados en la cara interna de las bandas de refuerzo apical que rodean el complejo faneroide por su parte exte- 

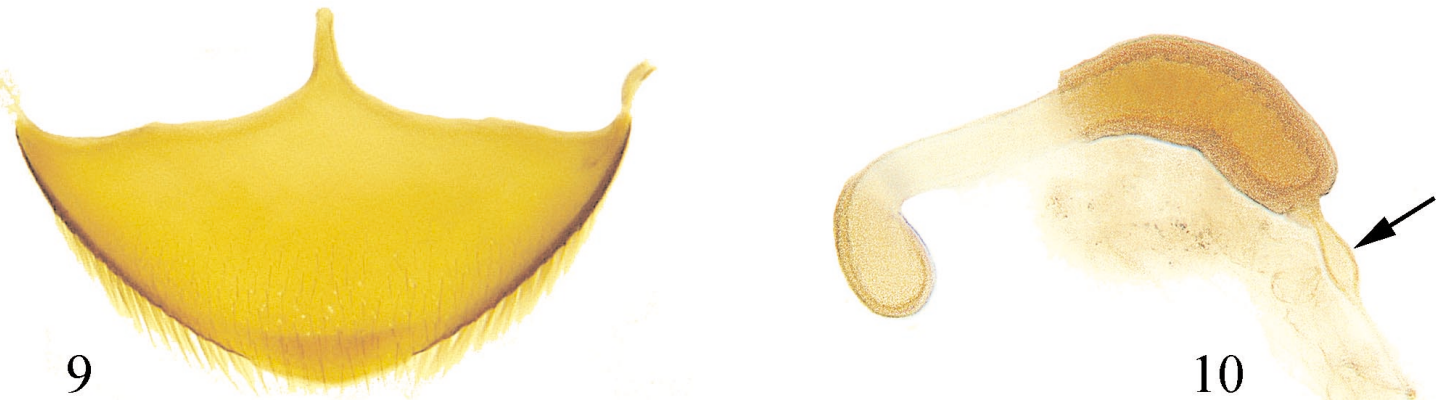

\section{$0,5 \mathrm{~mm}: 9$}
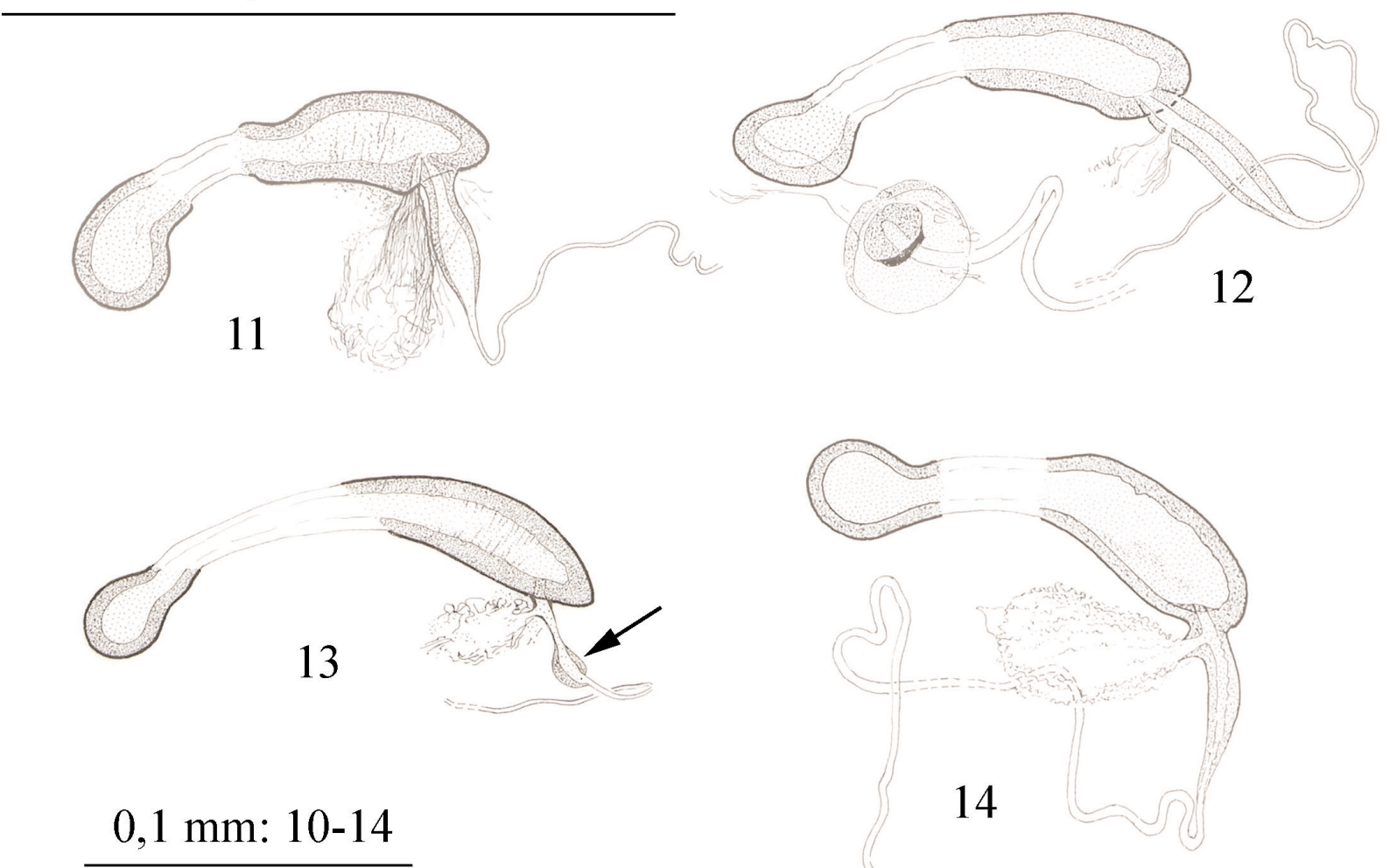

0,1 mm: $10-14$

14

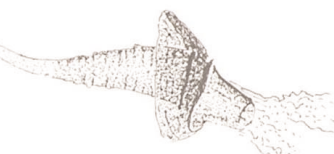

Figs. 9-14.- Octavo uroesternito abdominal femenino de 9) Bathysciola fadriquei n. sp. Espermateca de 10) B. fadriquei n. sp. 11) B. breuili. 12) B. diegoi. 13) B. obermaieri. 14) B. rugosa. La flecha indica el engrosamiento del conducto espermático localizado poco antes del punto de inserción con la espermateca.

Figs. 9-14. - Female $8^{\text {th }}$ abdominal urosternite of 9) Bathysciola fadriquei n. sp. Spermatheca of 10) B. fadriquei n. sp. 11) B. breuili. 12) B. diegoi. 13) B. obermaieri. 14) B. rugosa. The arrow marks the enlargement of the spermatic duct short before the insertion in the spematheca. 
rior (Fig. 4: PBRA); éstos son poco evidentes en B. breuili (Fig. 5), B. diegoi (Fig. 6) , B. obermaieri (Fig. 7) y B. rugosa (Fig. 8).

- En B. fadriquei n. sp. se encuentra un lóbulo redondeado asociado a la pieza basal del complejo faneroide (Fig. 4: LECF) que también se encuentra en B. obermaieri (Fig. 7), aunque en ésta no es redondeado sino puntiagudo; esta estructura no existe en ninguna de las otras tres especies, B. breuili (Fig. 5), B. diegoi (Fig. 6) y B. rugosa (Fig. 8).

- La existencia de unas faneras apicales del complejo faneroide muy grandes, triangulares y fuertemente esclerotizadas (Fig. 4: PACF) diferencia a $B$. fadriquei $\mathbf{n}$. sp. de todas las especies, no sólo de las cuatro ibéricas $(B$. breuili, $B$. diegoi, $B$. obermaieri y $B$. rugosa) en las que estas estructuras son pequeñas e hialinas, sino también de las norpirenaicas.

- Bathysciola breuili tiene el metafémur del macho con espina femoral, mientras que $B$. fadriquei n. sp., y las otras tres, $B$. diegoi, $B$. obermaieri y B. rugosa, lo tienen simple.

- En B. fadriquei n. sp. el conducto espermático tiene un engrosamiento poco antes del punto de inserción con la espermateca (Fig. 10), igual que se encuentra en $B$. obermaieri (Fig. 13); en las otras tres especies, B. breuili (Fig. 11), B. diegoi (Fig. 12) y B. rugosa (Fig. 14), está exactamente situado en el punto de inserción.

DisTRIBUCIÓN GEOGRÁFICA. Las Bathysciola del grupo schiodtei se distribuyen por los Pirineos y habitan ambas vertientes de la mitad occidental del macizo; también se han dispersado hacia el norte siguiendo el curso del río Garona hasta cerca de Burdeos, y se sabe de una especie que habita en la región de Calvados, al norte de Francia. Las especies ibéricas colonizan los relieves vasco-navarros dónde existe una rica y bien conocida fauna de Leptodirini; también los territorios de la vertiente sur de la mitad occidental de los Pirineos, desde el valle de Tena hacia el oeste, donde parecen ser los únicos Leptodirini -exceptuando Speonomus ere Escolà y Fresneda, 2001, de Peña Forca-Alanos en el valle de Echo-. El hallazgo de Bathysciola fadriquei $\mathbf{n}$. sp. hace patente el conocimiento fragmentario que existe de la fauna de Leptodirini hipogeos de esta región de Pirineos dónde sólo se han hecho muestreos muy localizados y esporádicos.

Dada la confusión que ha existido en este grupo (véase Fresneda \& Salgado, 2006) sólo se consignan los datos de distribución comprobados personalmente por los autores.
- Bathysciola breuili está distribuida por las provincias de Álava -Murúa, Peña Gorbeia-, Guipúzcoa -Rentería, Cueva de Landarbaso (localidad típica) - y Navarra - dato inédito: Garralda, Sima de Garralda, 26-V-2004, Faille leg.-.

- Bathysciola diegoi se conoce de Navarra: Arive -Cueva de Mendia Landa (localidad típica)-; datos inéditos: Villanueva de Aézkoa, Sima Hermanas Elukiate, y bajo piedras en el hayedo donde se encuentra la boca de entrada de la cavidad, 28-VIII-2004, Faille leg.

- Bathysciola fadriquei n. sp. ha sido encontrada en dos cavidades situadas en la Sierra de Bernera (macizo del Bisaurín) en Huesca: Jaca, Aragüés del Puerto, Cueva de Lizara -localidad típica- y Espelunga de Mediodia.

- Bathysciola obermaieri se distribuye por la provincia de Huesca: Biescas - Cueva de la Ermita de Santa Elena (localidad típica)- y Villanúa -Cueva de las Guixas, Cueva Nueva y Cueva del Rebeco-.

- Bathysciola rugosa habita en la provincia de Navarra: Alsásua (localidad típica) - Cueva de Orobe-, Abaurrea Alta -Cueva del Puente-, Espinal, Sierra de Aralar, Villanueva de Aézkoa -Cueva de Artekia y Sima Hermanas Elukiate (dato inédito, 28-VIII-2004, Faille leg.)-y Sierra de Urbasa -Bidoiza (dato inédito, 30-VII-2006, Fresneda leg.)-.

\section{Clave de especies ibéricas}

1. Saco interno del edeago con las faneras basales del complejo faneroide rectas y el extremo distal pequeño, mientras que la mitad basal está engrosada (Figs. 6,7) .......... 2

- Saco interno del edeago con las faneras basales del complejo faneroide muy dobladas por la mitad, formando un ángulo recto o un poco agudo (Figs. 4, 5, 8) .................. 3

2. Longitud: $2,00-2,50 \mathrm{~mm}$. Con los élitros más deprimidos en el tercio apical y el contorno marcadamente atenuado hacia atrás. El saco interno del edeago (Fig. 7) con la fanera superior del complejo faneroide formada por una placa triangular con dos márgenes engrosados; la fanera intermedia prolongada y con el margen superior engrosado de modo que sobresale hacia la línea axial del saco; las faneras ventrales y laterales más gráciles y poco esclerotizadas. La espermateca es más larga y delgada, con el lóbulo apical proporcionalmente pequeño (Fig. 13)

B. obermaieri Bolívar, 1918

- Longitud: 1,70-2,00 mm. Con los élitros más convexos y el contorno regularmente oval. El saco interno del edeago (Fig. 6) con la fanera superior del complejo faneroide formada por una simple placa triangular poco esclerotizada; la fanera intermedia con forma de diente triangular y también formada por una placa plana; las faneras ventrales y latera- 
les muy esclerotizadas y robustas. La espermateca es más corta y robusta, con el lóbulo apical proporcionalmente más grande (Fig. 12) …... B. diegoi Salgado y Fresneda, 2001

3. Metafémures del macho provistos de un marcado dentículo en su margen posterior ............ B. breuili Bolívar, 1921

- Metafémures del macho simples, desprovistos de espina femoral

4. Las faneras basales del complejo faneroide del saco interno del edeago van acompañados de unos lóbulos exteriores; las faneras apicales son muy grandes, triangulares y están fuertemente esclerotizadas; entre las bandas de refuerzo se encuentra un túbulo y en la cara interna de su parte inferior existen sendos pedúnculos de gran tamaño (Fig. 4) B. fadriquei n. sp.

- Las faneras basales del complejo faneroide del saco interno del edeago no van acompañadas de lóbulos exteriores; las faneras apicales son pequeñas, triangulares y están débilmente esclerotizadas; entre las bandas de refuerzo no se encuentra ningún tipo de estructura y en la cara interna de su parte inferior se encuentran unos finos pedúnculos débilmente esclerotizados (Fig. 8)

B. rugosa (Sharp, 1872)
Giachino, P. M., 1998. Una nuova Bathysciola del Caucaso (Coleoptera Cholevidae: Leptodirinae). Annali del Museo civico di Scienze Naturali di Brescia, 31 [1995]: 105-112.

JEANNEL, R., 1910. Essai d'une nouvelle classification des Silphides cavernicoles. Archives de Zoologie expérimentale et générale, 45(1): 1-48.

JeAnnel, R., 1924. Monographie des Bathysciinae (Col. Catopidae). Archives de Zoologie expérimentale et générale, 63(1): 1-436.

Newton, A. F., 1998. Phylogenetic problems, current classification and generic catalogue of world Leiodidae (including Cholevinae). In: Giachino, P. M. y Peck, S. B. (eds.). Phylogeny and evolution of subterranean and endogean Cholevidae (= Leiodidae Cholevinae). Proceedings of a Symposium XX International Congress of Entomology, Firenze, 1996: 41-178.

Perreau, M., 2000. Catalogue des Coléoptères Leiodidae Cholevinae et Platypsyllinae. Mémoires de la Société entomologique de France, 4: 1-460.

\section{AGRADECIMIENTOS}

A los queridos compañeros F. Fadrique (Hospitalet de l'Infant, Tarragona) y Dr. A. Faille (Paris, Francia), por confiar para su estudio los Leiodidae que, con admirable constancia, recolectan en medio subterráneo. Al Dr. I. Ribera por su ayuda en la traducción al inglés del resumen y los pies de figura y al Dr. J.M. Salgado por la lectura crítica del manuscrito, cuyas correcciones y sugerencias han contribuido substancialmente. Este trabajo se ha beneficiado en parte de una ayuda del proyecto «Fauna Ibérica» (REN 2001-1956-C17-09); algunas de las ilustraciones que se reproducen pertenecen a este proyecto.

\section{Bibliografía}

Fresneda, J. y Salgado, J. M., 2000. Revisión de los géneros de Leptodirinae de la Sección Speonomus del sur de los Pirineos. II: géneros Aranzadiella Español, 1972, Euryspeonomus Jeannel, 1919, Kobiella Español y Bellés, 1980 y Speocharidius Jeannel, 1919 (Coleop-tera, Cholevidae). Mémoires de Biospéologie, 27: 41-52.

Fresneda, J. y SAlgado, J. M., 2006. The genus Bathysciola Jeannel, 1910 in the Iberian Peninsula and Pyrenees. Taxonomic revision of the sections IV, VI and VII (Jeannel, 1924) (Coleoptera, Cholevidae, Leptodirinae). Graellsia, 62(1): 25-74.

Fresneda, J., Salgado, J. M. \& Ribera, I., 2007. Phylogeny of Western Mediterranean Leptodirini, with an emphasis on genital characters (Coleoptera: Leiodidae: Cholevinae). Systematic Entomology, 32: 332-358. 